\title{
Fluorogenic Protein Probes with Red and Near-Infrared emission for Genetically Targeted Imaging
}

\author{
Sylvestre P. J. T. Bachollet, $\left[{ }^{[a]}\right.$ Cyril Addi, ${ }^{[b]}$ Nicolas Pietrancosta, ${ }^{[a],[c]}$ Jean-Maurice Mallet ${ }^{[a]}$ and Blaise \\ Dumat ${ }^{*[a]}$
}
[a] Dr. S. P. J. T. Bachollet, Dr. N. Pietrancosta, Dr. J.-M. Mallet and Dr. B. Dumat
Laboratoire des biomolécules, LBM, Département de chimie, École normale supérieure, PSL University, Sorbonne Université, CNRS, 75005 Paris, France. E-mail: blaise.dumat@ens.psl.eu
[b] Dr. C. Addi
Membrane Traffic and Cell Division Lab, Cell Biology and Infection Department, Institut Pasteur, CNRS UMR3691, Sorbonne Université, 75005 Paris.
[c] Dr. N. Pietrancosta.
Neuroscience Paris Seine - Institut de Biologie Paris Seine (NPS - IBPS) INSERM, CNRS, Sorbonne Université, Paris, France.

Supporting information for this article is given via a link at the end of the document.

\begin{abstract}
Fluorogenic probes are important tools to image proteins with high contrast and no wash protocols. In this work, we rationally designed and synthesized a small set of four protein fluorogens with red or near-infrared emission. The fluorophores were characterized in the presence of albumin as a model protein environment and exhibited good fluorogenicity and brightness (fluorescence quantum yield up to $36 \%$ ). Once conjugated to a haloalkane ligand, the probes reacted with the protein self-labeling tag HaloTag with a high fluorescence enhancement (up to 156-fold). The spectroscopic properties of the fluorogens and their reaction with HaloTag were investigated in vitro experimentally and with the help of molecular dynamics. The two most promising probes, one in the red and one in the near-infrared range, were finally applied to image the nucleus or actin in live-cell and in wash-free conditions using fluorogenic and chemogenetic targeting of HaloTag fusion proteins.
\end{abstract}

\section{Introduction}

Genetically encoded fluorescent reporters have become major tools for studying cellular functions such as protein locations, concentrations, and protein-protein interactions. ${ }^{[1]}$ Starting from the pioneering work on the Green Fluorescent Protein (GFP), biochemists have developed a palette of fluorescent proteins (FPs) with enhanced brightness and stability and a variety of color. [2] More recently, molecular tagging of recombinant proteins using hybrid chemogenetic systems has garnered significant interest. ${ }^{[3]}$ Several strategies have been developed from small peptide tags, with the groundbreaking $\mathrm{FIAsH}^{[4]}$ and $\mathrm{ReAsH}^{[5]}$ systems to the highly selective protein tags SNAP ${ }^{[6]}$, CLIP-tag ${ }^{[7]}$ or HaloTag. ${ }^{[8]}$ Protein tags usually involve the fusion of a protein of interest with a modified enzyme able to selectively react with a substrate to form a covalent complex. By conjugating said substrate to various molecular tools, this technology has found applications in protein purification, manipulation and imaging. ${ }^{[9]}$ As an alternative to fluorescent proteins, this post-expression labeling strategy holds several advantages for bioimaging: (1) it does not require the maturation of a protein chromophore (2) it affords a better temporal control over the labeling (allowing pulse-chase experiments for instance) and (3) by conjugating them to the proper ligand, a variety of fluorescent reporters or even fluorescent sensors can be targeted to the same tag. Protein tags are thus highly valuable tools to perform cellular imaging by combining the genetic targeting ability of FPs with the diversity of fluorescent molecular probes. HaloTag has emerged as one of the most useful technology: as a modified haloalkane dehalogenase enzyme, it reacts selectively with a chemically simple halogenoalkane ligand to form a covalent complex with very fast reaction kinetics. It has been used to image proteins with a variety of fluorescent reporters (including some commercial ones) or to target biosensors such as $\mathrm{Ca}^{2+}$ or $\mathrm{Zn}^{2+}$ probes. ${ }^{[8-11]}$

Even with an efficient targeting strategy, one still has to get rid of the unbound dyes that may interfere with the experiments by emitting an off-target signal, which can be a critical flaw in experiments where the excess dye cannot easily be washed away. The use of fluorogenic probes that specifically light-up upon binding to their target but are otherwise dark circumvents this issue since unbound dyes will remain undetected, thus ensuring high contrast images. ${ }^{[12-14]}$ The fluorogenic targeting of protein self-labeling tags is thus a very useful strategy to image protein with high contrast and in no-wash conditions. Smart designs have been devised to develop fluorogenic protein probes, by combining fluorophore-quencher pairs that are separated upon reaction with the $\operatorname{tag}^{[15]}$ or by using a two-step labeling protocol involving a fluorogenic reaction such as tetrazine ligation. ${ }^{[16-18]}$ A simpler strategy is to use intrinsically fluorogenic probes that change their emissive properties upon reaction or binding with the cognate host. Such fluorogenic structures have been reported for protein self-labeling tags, involving mostly two approaches: (1) the isomerization of SiRhodamines between a dark and a fluorescent form, the latter being favoured in the hydrophobic environment of a protein ${ }^{[19,20]}$ or (2) conjugated donor-acceptor structures with solvatochromic or molecular rotor properties that are intrinsically sensitive to the viscosity and/or polarity of the environment. Molecular rotors are flexible push-pull molecules and are characterized by a chargetransfer singlet state that leads to non-radiative decay through rotation around the donor-acceptor bond. In a constrained environment, such as a protein binding pocket, and often in combination with hydrophobic interactions, the radiative decay is restored, leading to a large increase of the fluorescence intensity. ${ }^{[21,22]}$ Based on this strategy, some fluorogenic dyes for Halotag and SNAP-tag have been built on the solvatochromic naphthalimide ${ }^{[23]}$ or benzothiadiazole $e^{[24,25]}$ scaffolds, or on the classical molecular rotor 9-(2-carboxy-2-cyanovinyl)julolidine (CCVJ). ${ }^{[26,27]}$ These fluorophores display greenish fluorescence emission and very few examples exist with far-red or nearinfrared (NIR) emissions, despite the fact that it is a highly desirable feature for in vivo imaging. Zhang and coworkers tested an analog of the chromophore from the red fluorescent 
protein Kaede as a fluorogenic probe targeting SNAP-tag, ${ }^{[28]}$ and, for Halotag, a series of red-emitting pyridinium styryl dyes called "channel dyes" have been described by Kool and coworkers. ${ }^{[29]}$ In this latter report, it is assumed that the insertion of a flexible styryl dye (a typical molecular rotor) within the narrow channel of the HaloTag binding pocket results in a constrained planar form with an increased fluorescence. The probes have however only been used in live imaging of bacteria and the use of cationic pyridinium may cause selectivity conflicts, since it has been reported for similar cationic dyes to accumulate in the mitochondria of eukaryotic cells. ${ }^{[30,31]}$

Si-Rhodamine-based fluorogens benefit from the very good photophysical properties of the scaffold (far-red emission, high brightness and photostability). Recent derivatives developed by the team of Kai Johnsson also display considerably enhanced

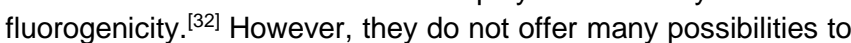
diversify their properties. Molecular rotors, due to their very generic design principles, encompass a large variety of structures and constitute a wider chemical space that can be explored to develop novel fluorogenic probes for protein tags with diverse photophysical and biochemical properties. In this work, we have thus set out to develop a series of efficient long wavelength HaloTag fluorogens for targeted live-cell imaging. A first series of fluorogen structures was characterized using Bovine Serum Albumine (BSA) as a model protein and the probes were subsequently coupled to a Haloalkane ligand in order to selectively react with HaloTag. The resulting targeted probes were studied in vitro, and then applied to biological imaging in live cells transfected with HaloTag fusion proteins.

\section{Results and Discussion}

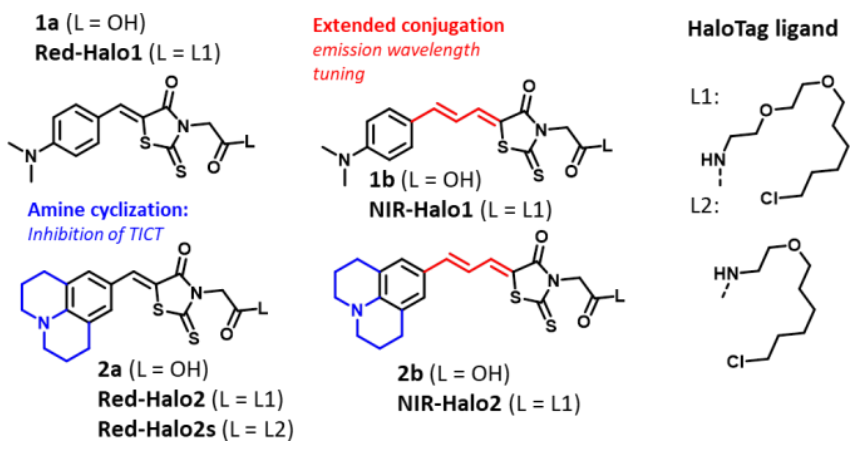

Figure 1. Structures of the fluorogenic protein probes for BSA and Halotag.

Probe design. To achieve fluorogenicity, the probes were rationally designed as push-pull molecular rotors with a dialkylaminobenzene electron-donating group and a rhodanine acceptor group (Figure 1). Two different lengths of $\pi$-conjugation were used to achieve red and NIR emission. In addition to the usual dimethylaminobenzene donor, julolidine derivatives were also synthesized. In addition to the rotation around the double bond that is expected to be inhibited upon binding to a target protein, the rotation around the aniline bond offers an additional non radiative deactivation pathway via the formation of a twisted intramolecular charge transfer (TICT) state. The cyclization of the amine with a julolidine is expected to inhibit the formation of the TICT state, thus leading to higher fluorescence brightness. ${ }^{[33]}$ Rhodanine has been chosen since it is neutral, thus preventing non-specific electrostatic interactions, and the carboxylic acid function allows facile further conjugation to a selective protein ligand. Very close derivatives of $\mathbf{1 a}$ and $\mathbf{1} \mathbf{b}$ have previously been reported and display solvatofluorochromic and viscositysensitive emissions. ${ }^{[31,34]}$ The rhodanine benzylidene conjugates also resemble the structure of the GFP chromophore and a similar scaffold can be found, for instance, on previously reported protein or RNA aptamers fluorogens, which makes it overall promising for our purposes. ${ }^{[35-37]}$ The probes were obtained in a straightforward synthesis, using the Knoevenagel condensation of rhodanine-3-acetic acid on the corresponding starting aldehydes, followed by an amide coupling with a halogenoalkane chain (Scheme S1).

Interaction with BSA. In order to first characterize the optical properties of the fluorophore moiety in the most general setting (i.e. without a targeting ligand), we have studied the intermediate probes $\mathbf{1} \mathbf{a}-\mathbf{b}$ and $\mathbf{2} \mathbf{a}-\mathbf{b}$ using bovine serum albumin (BSA). BSA is a serum transport protein that contains several sites able to bind a wide variety of organic and inorganic species. ${ }^{[38-40]}$ In particular, it has been reported to bind fluorogenic triphenylamine derivatives of our compounds. ${ }^{[41]}$ As such, it is a good generic model to easily and rapidly assess the fluorogenic character of our probes in a protein environment regardless of the ligand. The optical properties of probes $\mathbf{1} \mathbf{a}-\mathbf{b}$ and $\mathbf{2} \mathbf{a}-\mathbf{b}$ were thus characterized in presence of BSA (Table 1 \& Figure 2). The fluorescence of the probes is almost negligible in water but they became highly emissive in presence of BSA with large enhancement of the fluorescence intensity by up to 280 -fold (Figure 2B). Fluorimetric titrations of the probes with BSA can be fitted with a one-site binding model and the calculated dissociation constants $\mathrm{K}_{\mathrm{D}}$ are in the micromolar range (Figure $\mathrm{S} 1)$. This shows that the fluorogenicity originates from a binding equilibrium with albumin with a good affinity, leading to hydrophobic interactions and motion restrictions that increase the fluorescence quantum yield (Figure S1).

Table 1. Optical properties of compounds $\mathbf{1} \mathbf{a}-\mathbf{b}-\mathbf{2} \mathbf{a}-\mathbf{b}$ in presence of 20 equivalents of BSA

\begin{tabular}{cccccccc}
\hline & $\begin{array}{c}\lambda_{\text {abs }} \\
(\mathrm{nm})\end{array}$ & $\begin{array}{c}\varepsilon \\
\left(\mathrm{M}^{-1} \cdot \mathrm{cm}^{-1}\right)\end{array}$ & $\begin{array}{c}\lambda_{\mathrm{em}} \\
(\mathrm{nm})\end{array}$ & $\begin{array}{c}\Delta \lambda \\
(\mathrm{nm})\end{array}$ & $\Phi_{\mathrm{F}}$ & $\varepsilon \cdot \Phi_{\mathrm{F}}$ & $\mathrm{F} / \mathrm{F}_{0}$ \\
\hline 1a & 482 & 45000 & 563 & 77 & 0.034 & 1775 & 26 \\
2a & 516 & 58300 & 590 & 71 & 0.36 & 21000 & 287 \\
1b & 522 & 55600 & 650 & 122 & 0.029 & 1612 & 10 \\
2b & 568 & 26300 & 672 & 104 & 0.081 & 2130 & 79 \\
\hline
\end{tabular}

The compounds display broad emission spectra in the red (1a $\mathbf{2 a}$ ) and NIR range (1b, 2b) (Figure $2 \mathrm{~A}$ ) with large Stokes shifts. The cyclization of the donating amino group with a julolidine is accompanied by a red-shift $(20-40 \mathrm{~nm})$ of the absorption and emission wavelengths, in agreement with the stronger donating character of the julolidine, ${ }^{[42]}$ and by a large increase of the fluorescence quantum yield, thanks to the inhibition of the TICT state formation. ${ }^{[3]}$ The improvement of the quantum yield results in a much larger fluorescence enhancement in the presence of proteins (26-fold and 281-fold enhancement for $\mathbf{1 a}$ and $\mathbf{2 a}$ respectively, Figure $2 \mathrm{~B}$ ). The extension of the conjugation decreases the fluorescence quantum yield, as is often the case for red-shifted fluorophores due to the increased rotational freedom and lower energy difference between the ground and excited states. Cyclization of the electron donating amine in the extended structure results in similar increase of the quantum yield and red-shift of the emission wavelength between $\mathbf{1 b}$ and $\mathbf{2 b}$. Overall, compound $\mathbf{2 a}$ is thus the most promising probe, with 
a large fluorescence enhancement and an excellent brightness of $21000 \mathrm{M}^{-1} \mathrm{~cm}^{-1}$, on par with that of common red-emitting fluorescent proteins. ${ }^{[43]}$ Despite its lower quantum yield, $\mathbf{2 b}$ remains a very interesting fluorogen, considering its NIR emission centered on $672 \mathrm{~nm}$, with a good brightness and a 79. fold fluorescence enhancement in BSA. These results confirm the fluorogenic character of our fluorophores and suggest that, once properly targeted, they could be used for fluorogenic protein imaging in cells.
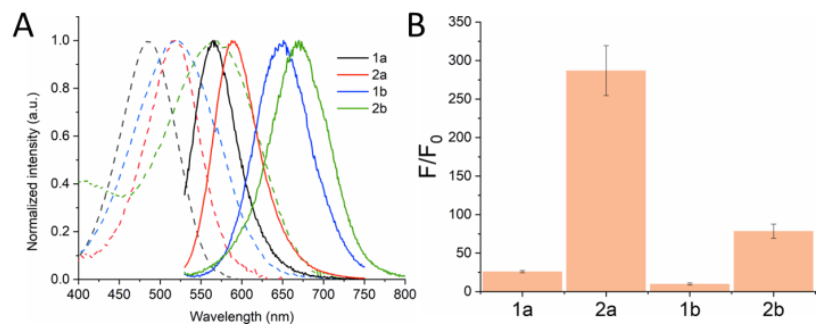

Figure 2. (A) Normalized absorption (dashed lines) and fluorescence emission (solid lines) spectra of compounds $1 \mathrm{a}-4 \mathrm{a}$ in presence of 25 eq of BSA. [dye] $=1 \mu \mathrm{M}$ in $\mathrm{pH} 7.4$ PBS (10 mM phosphate $+100 \mathrm{~mm} \mathrm{NaCl})$. (B) Maximum fluorescence enhancement F/F0 measured at saturation in BSA. [dye] $=1 \mu \mathrm{M}$, $[\mathrm{BSA}]=15-30 \mu \mathrm{M}$

Interaction with HaloTag in vitro. We have next conjugated our probes to a haloalkane linker in order to target HaloTag fusion proteins. The fluorogens were first coupled to the standard Halotag ligand reported in the literature to afford the Red-Halo and NIR-Halo compounds (Figure 1). Previous reports have shown that a shorter HaloTag ligand may increase the fluorogenicity through hydrophobic and cation- $\pi$ aromatic interactions or increased motion restriction. ${ }^{[24,29,44]}$ We have thus synthesized an analog of Red-Halo2 coupled to a shorter ligand chain with one ethylene glycol unit less: Red-Halo2s (Figure 1). We first assessed their optical properties in vitro using a standard GST-HaloTag (GST-HT) protein (Figure 3 and Table $\mathrm{S} 1$ ). Due to the addition of a lipophilic ligand, the HaloTag probes are less soluble in aqueous medium than their carboxylic acid counterpart, with broad and blue-shifted absorption bands in water showing signs of aggregation (Figure 3 ). In the presence of HaloTag, the lower-energy absorption bands are similar to those measured in BSA, which results in a red-shift of 50 to $70 \mathrm{~nm}$ of the absorption maxima of Red-Halo2 and NIRHalo1 compared to water. The emissive properties are also satisfyingly similar, with negligible fluorescence intensities in water and large enhancements of the red and NIR emissions upon reaction with the GST-HT protein (Figure 4A). The binding of Red-Halo2s to HaloTag is accompanied by a $48 \mathrm{~nm}$ hypsochromic shift of the fluorescence emission compared to water (from $625 \mathrm{~nm}$ to $577 \mathrm{~nm}$ ) that is not visible with the other probes. Due to its shorter ligand Red-Halo2s is more deeply embedded in the hydrophobic protein environment, thus causing this blue-shift while probes with a longer ligand are less affected. Fluorescence emission spectra of the probes bound to HaloTag are also narrower than in water which is evidence of the structural constraint imposed by the protein on the molecular rotor, with fewer vibrational levels for the bound form. The fluorescence of $2 \mathbf{a}$ was also measured in presence of GST-HT as a negative control and it displays no enhancement of the fluorescence, showing that the fluorogenicity results from a specific reaction between the probes and their target HaloTag to form a covalent fluorescent complex.
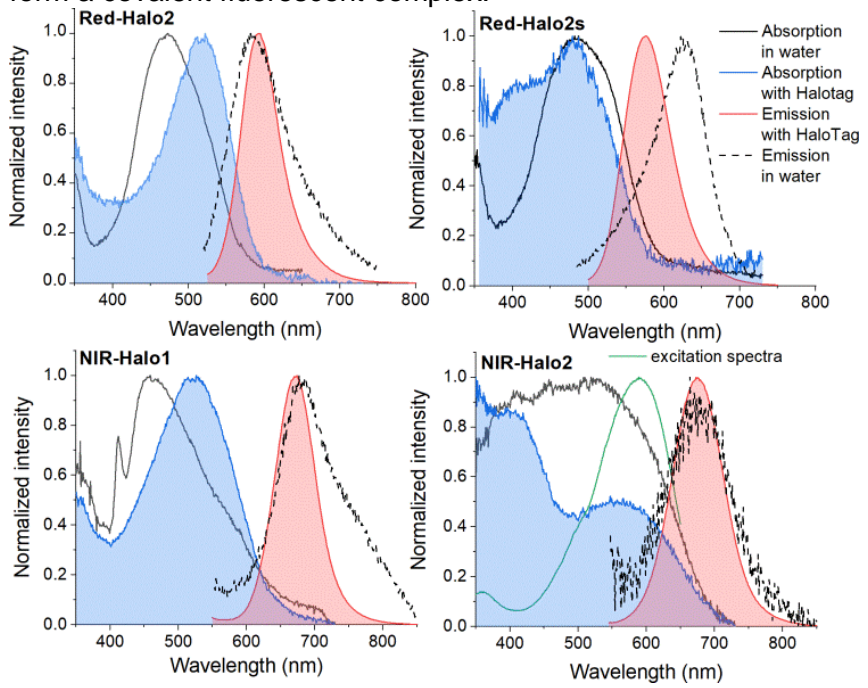

Figure 3. Spectral properties of the fluorogenic HaloTag dyes in water and after reaction with HaloTag. Normalized absorption (black full line) and emission (black dashed line) spectra in water, normalized absorption (blue) and emission (red) spectra in presence of HaloTag. For NIR-Halo2 is also displayed the excitation spectrum in green. $[$ dye $]=1 \mu \mathrm{m}$, [HaloTag $]=1.3 \mu \mathrm{M}$.

By following the evolution in time of the fluorescence intensities of our probes upon reaction with HaloTag, we were able to assess the relative reaction kinetics as well as to measure the fluorescence enhancement factors of each compounds (i.e. the ratio between the fluorescence intensity upon complete reaction with HaloTag and the initial fluorescence intensity) (Figure 4). In agreement with the design rationale and the properties in BSA, starting from the Red-Halo1 structure, the cyclization of the amine elicited a net improvement of the emissive properties, with a fluorescence quantum yield of $17 \%$ and a fluorescence enhancement of 156 for Red-Halo2 bound to HaloTag (Figure 4A). The photophysical properties of Red-Halo1 were thus not studied in further details. The shorter HaloTag ligand of RedHalo2s affords a slightly lower enhancement factor of 110, but with a higher fluorescence quantum yield (Table S1). The binding is however associated with a drop in absorptivity that results in a fluorescence brightness about 2.6-fold lower than that of Red-Halo2. This hypochromism, associated with a blueshift of the absorption band, and with the presence of a shoulder peak around $400 \mathrm{~nm}$, suggests that the insertion of the fluorophore unit within the HaloTag binding pocket induces structural constraints that partially break the electronic conjugation. The speed of the reaction of Red-Halo2s with HaloTag is also the lowest of all the probes (Figure 4B). The HaloTag ligand has been designed to fit the protein binding pocket and, presumably, the presence of bulky groups at the end of a shortened ligand sterically hinders the reaction. Overall, the use of the well-documented standard HaloTag ligand seems preferable with our structures since it results in higher fluorescence brightness and faster reaction.

In the NIR-Halo series, the dimethylamino compound NIR-Halo1 displays a similar fluorescence enhancement and a higher brightness than its julolidine counterpart, which contradicts the rational design and the properties observed in BSA for $\mathbf{1 b}$ and $\mathbf{2 b}$ (Figure 4A and Table S1). The absorption spectra of NIR- 
Halo2 shows, in addition to the lower energy absorption band centered on $565 \mathrm{~nm}$, a main absorption band centered on 408 $\mathrm{nm}$. This higher energy band does not contribute to the fluorescence emission according to the excitation spectra (Figure 3). Since we do not observe a decrease of fluorescence over time (Figure 4B), this form is not the result of degradation and it is more likely that the reaction with HaloTag produces two conformations, including one where NIR-Halo2 is forced into a lesser conjugated, non-fluorescent form. This results in a low brightness and the high fluorescence enhancement factor recorded for NIR-Halo2 is only due to a very low initial intensity $\mathrm{F}_{0}$. On the other hand, NIR-Halo1 displays a quantum yield of $5.3 \%$ and a satisfactory brightness for a NIR-emitting compound Overall, considering brightness and contrast, Red-Halo2 and NIR-Halo1 are very promising fluorogenic HaloTag probes. NIRHalo1 reacts with HaloTag within 15 minutes, while the reaction with Red-Halo2 is essentially complete after 60 minutes (Figure $3 \mathrm{~B})$. This large difference in reaction rate between two probes with the same ligand is rather surprising and may be explained by different aggregation states, since aggregation will slow the reaction with the HaloTag.
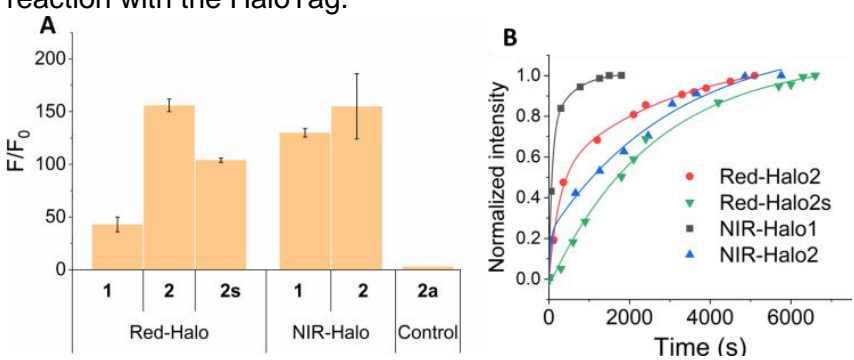

Figure 4. (A) Fluorescence enhancement $F / F_{0}$ of the probes upon reaction with GST-HT. Intermediate $\mathbf{2 a}$ was also studied as a negative control (B) Time-course measurement of the normalized integrated fluorescence intensity upon addition of GST-HT ( $\mathrm{T}=296 \mathrm{~K})$. Trend lines have been added only as a visual guide. [dye] $=1 \mu \mathrm{M},[$ Halotag $]=1.3 \mu \mathrm{M}$.

Molecular dynamics. We have then used molecular modeling to better understand the binding of the probes to HaloTag and the discrepancies observed in the experimental optical properties between NIR-Halo1 and NIR-Halo2. After solvation, a first energy minimization of the probes bound to HaloTag, we have performed a 3 ns equilibration with a 10 ps step, generating 300 conformations. We next made a cluster analysis to isolate the main conformations, yielding 2 clusters for RedHalo2s (50\% and 50\%), 3 for NIR-Halo2 (36\%, 32\% and 32\%) and 4 for Red-Halo2 (31\%, 30\%, 23\% and 16\%) and NIR-Halo1 (42\%, 24\%, 23\% and 11\%). A representative conformation for each cluster is displayed on Figure S2 and Figure 5 highlights the conformation with minimal potential energy for each compound. For all compounds with a standard-length ligand, the fluorophore moiety is interacting only slightly, if not at all with the protein and remains largely exposed to the solvent and mobile, despite some hydrophobic interactions. The most recurring interaction for all the compounds is a $\pi-\pi$ interaction between the rhodanine cycle and a phenylalanine (F144). This may explain the lower brightness in HT compared to BSA since the molecular rotors are not properly shielded from the aqueous medium or constrained. Nonetheless, this configuration with no specific binding of the fluorophore moiety to the protein is still sufficient to elicit a large fluorescent intensity enhancement, which suggests that, with the proper ligand, our structures may be used as fluorogenic probes for a variety of proteins with little or no additional optimization. With its shorter ligand, RedHalo2s is partly buried within the ligand binding pocket and interacts more strongly with the protein. It has one cluster of planar conformations and a second where the two cycles are twisted by almost $90^{\circ}$, thus completely breaking the conjugation (Figure 5). This last conformation may be at the origin of the shoulder absorption band around $400 \mathrm{~nm}$ measured for RedHalo2s. By hindering the electronic conjugation, the additional constraint on the rotor structure is thus, in this particular case, detrimental to the fluorescence emission as we have measured experimentally (vide supra).
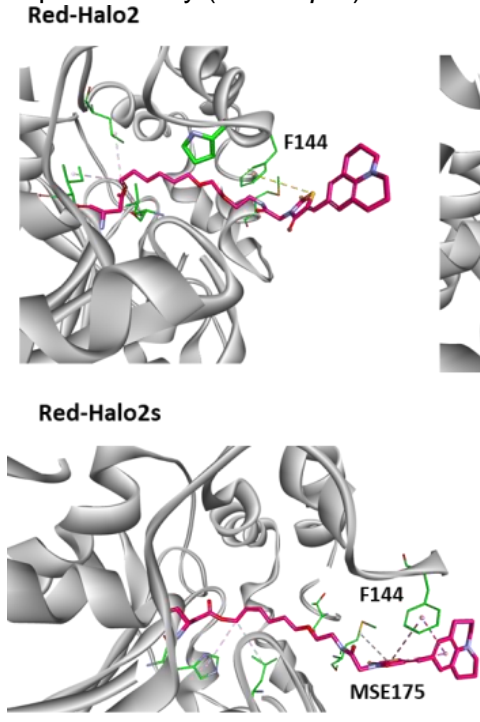

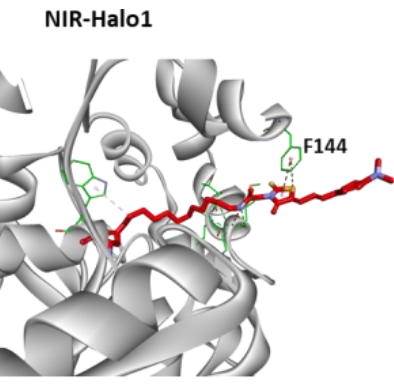

NIR-Halo2

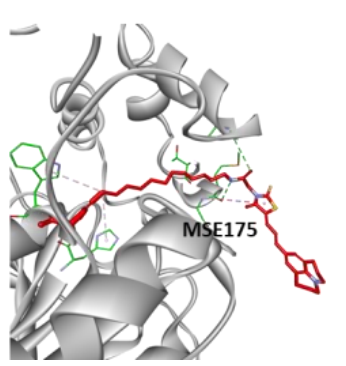

Figure 5. Representative structures of the conformation clusters with minimal potential energy of Red-Halo2, Red-Halo2s, NIR-Halo1 and NIR-Halo2 in HT calculated by molecular dynamics. Dashed lines show interactions with protein residues.

As expected with the increase of chain length, the flexibility of NIR-Halo1 and NIR-Halo2 is much higher than that of RedHalo2 or Red-Halo2s, with a RMSD ${ }_{\max }$ from reference pose of 8.54 and 19.87 versus 2.86 and 3.29 respectively (Figure S2). The four clusters of NIR-Halo1 all exhibit planar and conjugated structures. On the other hand, while two conformation clusters of NIR-Halo2 are planar, in the third one, the m-electron systems of the rhodanine and julolidine cycles are not conjugated with each other. This may explain the large absorption band of NIR-Halo2 around $400 \mathrm{~nm}$ corresponding to a lesser conjugated conformation and why NIR-Halo1 unexpectedly works better in $\mathrm{HT}$ than its julolidine counterpart.

Live cell imaging. Red-Halo2 and NIR-Halo1 were applied in live imaging of transfected Hela or HEK-293T cells expressing either HaloTag fused to a triple repeat of a nuclear localization sequence (PKKKRKV) ${ }^{[45]}$ Halo-NLS(3X) or HaloTag fused to the lifeact peptide Halo-Lifeact for actin imaging. ${ }^{[46]}$ Cells were incubated with $0.5 \mu \mathrm{m}$ of dye for 30 to 60 minutes and then imaged directly under a confocal microscope without performing washing steps or changing the medium. The cells stay alive after treatment and remained apparently unaffected even after longer incubation times (up to three hours). For both probes and in both cell lines, we observed a selective staining of the target 
structures as evidenced by comparison with non-transfected cells (Figure 6, Figure S3). Although the NLS peptide is expected to localize in the nucleoplasm, ${ }^{[47]}$ the Halo-NLS(3X) fusion protein shows a larger accumulation in the nucleoli, with a
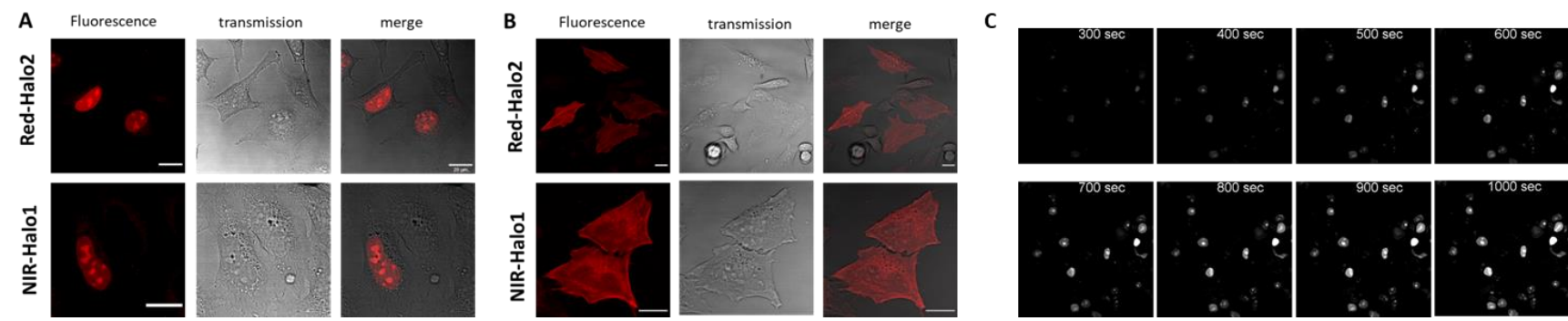

Figure 6. Live confocal microscopy imaging of Hela cells expressing either the Halo-NLS(3X) (A) or the Halo-lifeact (B) proteins and incubated with $0.5 \mu \mathrm{M}$ of compound Red-Halo2 or NIR-Halo1. [dye] $=0.5 \mu \mathrm{M}$, incubation $30-60$ minutes. $\lambda$ exc $=514 \mathrm{~nm}$, collection: $520-790 \mathrm{~nm}$. Scale bar $=20 \mu \mathrm{m}$. $(\mathrm{C})$ Time-course imaging of HEK-293T live cells transfected with Halo-NLS(3X) and incubated with $0.5 \mu \mathrm{M}$ of Red-Halo2

A time-course measurement of the fluorescence of HEK-293T cells transfected with Halo-NLS(3X) shows that the cell permeation and reaction with HaloTag is complete after ca. 15 minutes (Figure 6C and supplementary movie). The faster reaction rate than that observed in cuvette can be explained by the higher temperature and/or the different stoichiometry. Quantitative comparison between the two probes is difficult since the intensity strongly depends on the expression levels of the fusion protein, even between different cells within the same sample (see also Figure S4 for wider field of view images). However, both probes yield bright and contrasted images in agreement with the high fluorescence enhancements recorded in vitro. A faint non-specific signal can nonetheless be recorded in what appears to be the endoplasmic reticulum (ER), a localization that is consistent with previous observations made for similar untargeted structures. ${ }^{[31]}$ This signal can however be easily discriminated from the brighter specific emission of the probes bound to HaloTag and it does not hamper targeted imaging even in wash-free conditions (Figure $6 \mathrm{~A}$ and $6 \mathrm{~B}$ ). It could however be useful in future developments to perform further structure optimization to minimize the unspecific signal, especially to image low-abundant proteins.

\section{Conclusion}

In conclusion, we have developed a series of protein probes displaying bright emissions in the red and NIR range and high fluorogenicity in the presence of BSA. The fluorogens were then coupled to a Halogenoalkane ligand for the fluorogenic targeting of HaloTag fusion proteins. The targeted probes were successfully applied to the wash-free imaging of the nucleus and actin in live cells. The simple push-pull structures of our compounds provide a basis for further structure optimization and variations. Modifications of the electron-donor or acceptor moieties may be explored in future developments to fine-tune the photophysical properties, enhance the signal-to-noise ratio or improve cell permeation and labeling kinetics. The comparison between BSA and HaloTag shows that the protein matrix influences the emissive properties but the main characteristics and in particular the high fluorogenicity are conserved in both proteins. Conjugated to the proper ligand, our bright fluorogens may prove useful to target a variety of proteins either for imaging applications or to monitor biomolecular interactions in vitro.

\section{Experimental Section}

Materials. Chemical reagents and solvents were purchased from SigmaAldrich or $\mathrm{TCl}$ and were used as received. Purified GST-Halotag protein (product \#G4491) was purchased from Promega Corp. (Madison, Wi, USA) at a concentration of $2.55-2.63 \mathrm{mg} / \mathrm{ml}$ depending on the lot number. HEK-293T (CRL-3216) and Hela (CCL-2) cells were obtained from the ATCC

Interaction with Bovine Serum Albumine (BSA). The fluorogenic dyes were dissolved in DMSO at a stock concentration of $1 \mathrm{mM}$. The titration of the probes by BSA was performed by adding increasing amounts of BSA in the working solution ( $1 \mu \mathrm{M}$ probe concentration in $1 \mathrm{~mL}$ of $10 \mathrm{mM}$ phosphate buffer $\mathrm{pH} 7.4$ containing $100 \mathrm{~mm}$ of $\mathrm{NaCl}$ ) and recording the fluorescence spectrum. The resulting titration curves were fitted to a onesite binding model using Origin Pro 9 software.

Interaction with HaloTag. The fluorogenic dyes were dissolved in DMSO at a stock concentration of $0.5 \mathrm{~mm}$. The interaction of the probes with Halotag was assessed by incubating the dye at $1 \mu \mathrm{M}(0.4 \mu \mathrm{L}$ of stock solution) concentration with $1.3 \mu \mathrm{M}$ (ca. $6 \mu \mathrm{L}$ of commercial stock solution) of protein in a $200 \mu \mathrm{L}$ working volume of $\mathrm{pH} 7.4$ Phosphate buffer (10 mM phosphate with $100 \mathrm{mM} \mathrm{NaCl}$ ). Fluorescence spectra were then recorded at regular time intervals over the course of the reaction. After complete reaction with Halotag, the absorption spectra of the protein-bound probes were also recorded.

Molecular dynamics. 3D models of HaloTag coupled to the fluorogen compounds were generated based on a $3 D$ structure of Halotag (pdb entry: 4KAF) and then refined by several cycles of minimization and equilibrated by molecular dynamics simulations (3-ns runs) using the CHARMm force field. Bonds involving hydrogen atoms were constrained using the SHAKE algorithm. ${ }^{[48]}$ The Ramachandran plot of the final 3D model showed 93\% (273/295) favored, 6\% (18/295) allowed, and 1\% (1 Glu, 1 Leu and 2 Gly) disallowed residues for NIRHalo2, and respectively, 92\% ( 272/295), 6\% (17/295), 2\% (6/295) for NIRHalo1, 91\% ( 267/295), $7 \%$ (22/295), $2 \%(6 / 295)$ for REDHalo2s, and 90\% ( $265 / 295), 8 \%$ (23/295), $2 \%(7 / 295)$ for REDHalo2 . A solvent box with a periodic boundary of $9 \AA$ was added to the model. Solvation was completed with $0.145 \mathrm{M} \mathrm{KCl}$ using the solvation protocol implemented in Discovery Studio (Dassault Systèmes BIOVIA, Discovery Studio, 2019, San Diego: Dassault Systèmes, 2019). 
Confocal microscopy. HEK-293T and Hela cells were grown overnight on 8-well polymer $\mu$ slides from Ibidi (\#1.5 polymer coverslip, tissue culture treated) at $50 \mathrm{k}$ to $100 \mathrm{k}$ cells/well in $300 \mu \mathrm{L}$ of culture medium (Gibco) supplemented with $10 \%$ fcs. Culture medium was DMEM for HEK-293T cells and MEM (supplemented with sodium pyruvate and nonessential aminoacids) for Hela cells. Cells were then transfected with the desired plasmid using Fugene 6 (Promega Corp.) according to the manufacturer's protocol. After 24 hours, the cells were incubated 30-60 minutes with the dyes and imaged live on a Zeiss LSM710 laser scanning confocal microscope equipped with a Plan apochromat 40X/1.4 NA objective. Images were acquired using Zen 2009 software and then processed using ImageJ. $\lambda_{\mathrm{exc}}=514 \mathrm{~nm}$, light collection: $520-797 \mathrm{~nm}$.

\section{Acknowledgements}

The authors would like to thank Marie-Aude Plamont for her assistance in cell culture and Dr. Arnaud Echard for kindly providing molecular biology reagents. This work was supported by the Agence Nationale de la Recherche (ANR-18-CE44-0006).

Keywords: Fluorogenic protein probes $•$ HaloTag •

Fluorescence imaging $\bullet$ molecular rotors

\section{References}

[1] B. N. G. Giepmans, S. R. Adams, M. H. Ellisman, R. Y. Tsien, Science 2006, 312, 217-24.

[2] R. N. Day, M. W. Davidson, Chem. Soc. Rev. 2009, 38, 2887-2921.

[3] S. Mizukami, Y. Hori, K. Kikuchi, Acc. Chem. Res. 2014, 47, 247256.

[4] B. A. Griffin, S. R. Adams, R. Y. Tsien, Science (80-. ). 1998, 281, 269-272.

[5] S. R. Adams, R. E. Campbell, L. a Gross, B. R. Martin, G. K. Walkup, Y. Yao, J. Llopis, R. Y. Tsien, J. Am. Chem. Soc. 2002, 124, 6063-76.

[6] A. Keppler, S. Gendreizig, T. Gronemeyer, H. Pick, H. Vogel, K. Johnsson, Nat. Biotechnol. 2003, 21, 86-9.

[7] A. Gautier, A. Juillerat, C. Heinis, I. R. Corrêa, M. Kindermann, F. Beaufils, K. Johnsson, Chem. Biol. 2008, 15, 128-136.

[8] G. V. Los, L. P. Encell, M. G. McDougall, D. D. Hartzell, N. Karassina, C. Zimprich, M. G. Wood, R. Learish, R. F. Ohana, M. Urh, D. Simpson, J. Mendez, K. Zimmerman, P. Otto, G. Vidugiris, J. Zhu, A. Darzins, D. H. Klaubert, R. F. Bulleit, K. V. Wood, ACS Chem. Biol. 2008, 3, 373-382.

[9] L. Xue, I. A. Karpenko, J. Hiblot, K. Johnsson, Nat. Chem. Biol. 2015, 11, 917-923

[10] E. Tomat, E. M. Nolan, J. Jaworski, S. J. Lippard, J. Am. Chem. Soc 2008, 130, 15776-15777.

[11] M. Best, I. Porth, S. Hauke, F. Braun, D.-P. Herten, R. Wombacher, Org. Biomol. Chem. 2016, 5606-5611.

[12] L. Jullien, A. Gautier, Methods Appl. Fluoresc. 2015, 3, 042007

[13] M. P. Bruchez, Curr. Opin. Chem. Biol 2015, 27, 18-23.

[14] E. Kozma, P. Kele, Org. Biomol. Chem. 2019, 17, 215-233.

[15] X. Sun, A. Zhang, B. Baker, L. Sun, A. Howard, J. Buswell, D. Maurel, A. Masharina, K. Johnsson, C. J. Noren, M. Q. Xu, I. R. Corrêa, ChemBioChem 2011, 12, 2217-2226.

[16] A. Wieczorek, P. Werther, J. Euchner, R. Wombacher, Chem. Sci. A. Wieczorek, P. Werthe 8, 1506-1510.

[17] G. Knorr, E. Kozma, J. M. Schaart, K. Németh, G. Török, P. Kele, Bioconjug. Chem. 2018, 29, 1312-1318.

[18] L. G. Meimetis, J. C. T. Carlson, R. J. Giedt, R. H. Kohler, R. Weissleder, Angew. Chemie - Int. Ed. 2014, 53, 7531-7534.

[19] J. B. Grimm, A. K. Muthusamy, Y. Liang, T. A. Brown, W. C. Lemon, R. Patel, R. Lu, J. J. Macklin, P. J. Keller, N. Ji, L. D. Lavis, Nat Methods 2017, 14, 987-994.

[20] V. Mueller, Z.-G. Luo, K. Johnsson, G. Lukinavičius, N. Olivier, C. Eggeling, K. Umezawa, T. Plass, C. Schultz, L. Reymond, I. R. Corrêa Jr, E. A. Lemke, G. Yang, P. Heppenstall, A. Honigmann, S. Manley, Nat. Chem. 2013, 5, 132-139.

[21] A. S. Klymchenko, Acc. Chem. Res. 2017, 50, 366-375.

[22] D. Su, C. L. Teoh, L. Wang, X. Liu, Y.-T. Chang, Chem. Soc. Rev. 2017, 46, 4833-4844.

[23] S. Leng, Q. Qiao, L. Miao, W. Deng, J. Cui, Z. Xu, Chem. Commun. 2017, 53, 6448-6451.
[24] Y. Liu, K. Miao, N. P. Dunham, H. Liu, M. Fares, A. K. Boal, X. Li, X. Zhang, Biochemistry 2017, 56, 1585-1595.

[25] X. Jiang, A. K. Boal, Y. Liu, M. Fares, N. P. Dunham, Z. Gao, X. Zhang, S. S. Bollinger, K. Miao, Angew. Chemie Int. Ed. 2017, 56, 8672-8676.

[26] W. T. Yu, T. W. Wu, C. L. Huang, I. C. Chen, K. T. Tan, Chem. Sci. 2016, 7, 301-307.

[27] X. Zhang, M. Fares, Y. Zhai, Y. Liu, K. Miao, Y. Li, Z. Gao, Bioconjug. Chem. 2017, 29, 215-224.

[28] K. H. Jung, M. Fares, L. S. Grainger, C. H. Wolstenholme, A. Hou, Y Liu, X. Zhang, Org. Biomol. Chem. 2019, 17, 1906-1915.

[29] S. A. Clark, V. Singh, D. Vega Mendoza, W. Margolin, E. T. Kool, Bioconjug. Chem. 2016, 27, 2839-2843.

[30] R. Chennoufi, H. Bougherara, N. Gagey-Eilstein, B. Dumat, E. Henry, F. Subra, F. Mahuteau-Betzer, P. Tauc, M.-P. TeuladeFichou, E. Deprez, Chem. Commun. 2015, DOI 10.1039/C5CC05970D

[31] C. Li, M. Plamont, I. Aujard, T. Le Saux, L. Jullien, A. Gautier, Org. Biomol. Chem. 2016, 14, 9253-9261.

[32] L. Wang, M. Tran, E. D’Este, J. Roberti, B. Koch, L. Xue, K. Johnsson, Nat. Chem. 2020, 12, 165-172.

[33] Z. R. Grabowski, K. Rotkiewicz, W. Rettig, Chem. Rev. 2003, 103, 3899-4031.

[34] E. D. Rekaï, J. B. Baudin, L. Jullien, I. Ledoux, J. Zyss, M. Blanchard-Desce, Chem - A Eur. J. 2001, 7, 4395-4402

[35] M. Pellerano, D. Naud-Martin, M. Peyressatre, C. Prével, M.-P. Teulade-Fichou, M. Morris, F. Mahuteau-Betzer, ChemBioChem 2016, 17, 737-744.

[36] M.-A. Plamont, E. Billon-Denis, S. Maurin, C. Gauron, F. M. Pimenta C. G. Specht, J. Shi, J. Quérard, B. Pan, J. Rossignol, K. Moncoq, N. Morellet, M. Volovitch, E. Lescop, Y. Chen, A. Triller, S. Vriz, T. Le Saux, L. Jullien, A. Gautier, Proc. Natl. Acad. Sci. 2016, 113, 497502.

[37] M. You, S. R. Jaffrey, Annu. Rev. Biophys. 2015, 44, 187-206.

[38] M. Dockal, D. C. Carter, F. Rüker, J. Biol. Chem. 1999, 274, 29303 10.

[39] V. S. Jisha, K. T. Arun, M. Hariharan, D. Ramaiah, J. Am. Chem. Soc. 2006, 128, 6024-5.

[40] S. Samanta, S. Halder, G. Das, Anal. Chem. 2018, 90, 7561-7568.

[41] B. Dumat, G. Bordeau, A. I. Aranda, F. Mahuteau-Betzer, Y. EI Harfouch, G. Metgé, F. Charra, C. Fiorini-Debuisschert, M.-P. Teulade-Fichou, Org. Biomol. Chem. 2012, 10, 6054.

[42] O. Kwon, S. Barlow, S. A. Odom, L. Beverina, N. J. Thompson, E. Zojer, J. L. Brédas, S. R. Marder, J. Phys. Chem. A 2005, 109, 9346-9352.

[43] N. C. Shaner, P. A. Steinbach, R. Y. Tsien, Nat. Methods 2005, 2, 905-9.

[44] M.-G. Kang, H. Lee, B. H. Kim, Y. Dunbayev, J. K. Seo, C. Lee, H.W. Rhee, Chem. Commun. 2017, 53, 9226-9229.

[45] D. Kalderon, B. L. Roberts, W. D. Richardson, A. E. Smith, Cell 1984, 39, 499-509.

[46] J. Riedl, A. H. Crevenna, K. Kessenbrock, J. H. Yu, D. Neukirchen, M. Bista, F. Bradke, D. Jenne, T. A. Holak, Z. Werb, M. Sixt, R. Wedlich-Soldner, Nat. Methods 2008, 5, 605-607.

[47] R. M. Martin, G. Ter-Avetisyan, H. D. Herce, A. K. Ludwig, G. Lättig Tünnemann, M. C. Cardoso, Nucleus 2015, 6, 314-325.

[48] G. Ciccotti, J. P. Ryckaert, Comput. Phys. Reports 1986, 4, 346392 
